Nevena P. Ceković ${ }^{*}$

УДК $811.131 .1 ' 243$

Nataša L. Janićijević

ДОИ https://doi.org/10.18485/analiff.2017.29.2.4

Università di Belgrado

Facoltà di filologia

\title{
SCUSA, SAI, TI DICO... GLI EQUIVALENTI SERBI DEI SEGNALI DISCORSIVI ITALIANI DI ORIGINE VERBALE
}

\begin{abstract}
In linea con la nostra ricerca precedente (Ceković \& Janićijević, in stampa), incentrata sui segnali discorsivi (SD) italiani formati da verbi di percezione visiva e uditiva (guardare, vedere, sentire, ascoltare) e la loro traduzione in serbo, il presente contributo si propone di indagare ulteriormente le corrispondenze funzionali tra l'italiano e il serbo, focalizzandosi su altre forme aventi l'origine verbale: i SD derivanti dal verbo sapere, dire e scusare. Nel contributo si descrivono, da un lato, le principali caratteristiche e funzioni dei SD in questione e, dall'altro, si individuano e analizzano le corrispondenti forme in serbo. Confrontando i SD italiani con le loro traduzioni in serbo, si riconferma la conclusione che gli equivalenti semantici non sempre sono anche gli equivalenti pragmatici e che la corrispondenza tra i SD nelle due lingue va sempre cercata a livello funzionale.
\end{abstract}

Parole chiave: segnali discorsivi italiani, equivalenti serbi, verbi, traduzione.

\section{Introduzione}

Come oggetto di studio i segnali discorsivi (SD) sono presenti sulla scena linguistica internazionale ed italiana a partire dagli anni Ottanta, sotto gli auspici di varie discipline (linguistica del testo, semantica, analisi della conversazione e del discorso, sociolinguistica, pragmatica, glottodidattica, linguistica cognitiva ed interazionale, linguistica dei corpora). Oggi si assiste ad un moltiplicarsi degli studi sul tema, fino al punto da considerarli parte di un mercato che annualmente produce decine di contributi pubblicati su varie riviste scientifiche (Fraser, 1998: 301). Il motivo per un tale interesse va cercato sicuramente nella mancata chiarezza ri-

n.cekovic@ffil.bg.ac.rs 
guardo lo status teorico dei SD: circolano almeno una ventina di etichette (in inglese, italiano e serbo, v. Ceković-Rakonjac, 2011), una varietà di definizioni, descrizioni e tassonomie delle loro molteplici funzioni pragmatiche e discorsive (cfr., ad esempio, Schiffrin, 1987; Fraser, 2006; Jucker \& Ziv, 1998; Blakemoore, 2002; Bazzanella, 1995, 2001, 2006, 2008; per una dettagliata descrizione v. Ceković, 2016).

È cresciuto di recente anche l'interesse per gli aspetti traduttivi e contrastivi di questo fenomeno tipico del parlato e così anche di quello italiano (cfr. Bazzanella, 1999; Bazzanella \& Borreguero Zuloaga, 2011; Bazzanella et al., 2007; Khachaturyan, 2010; Nigoević \& Bilić, 2009). Tuttavia, questo interesse è ancora privo di un carattere generale o comunque più comprensivo dei numerosi appartenenti di questa categoria funzionale, frequentissima nel parlato spontaneo, e rimane focalizzato su singole forme come diciamo o allora. Lo stesso vale anche per 1'offerta lessicografica, riguardante in particolare i dizionari bilingui e monolingui (cfr. Deanović \& Jernej, 1998; Klajn, 2011; Zingarelli, 1994; Pittàno, 1997; Duro, 2003), che solo recentemente include in modo più esplicito e sistematico le descrizioni funzionali di alcune di queste particelle conversazionali (Sabatini \& Coletti, 2004; Zingarelli, 2011).

In conformità alle nostre ricerche precedenti sul campo (Ceković \& Janićijević, in stampa sui verbi di percezione visiva e uditiva; Ceković \& Radojević, 2016 sul segnale allora) e tenendo conto di quelle altrui (Bazzanella, 1995; Khaciaturian, 2002 su diciamo; Bazzanella, 1995 su sai), abbiamo l'intenzione di indagare ulteriormente le corrispondenze interpretative tra l'italiano e il serbo, ponendo al centro della nostra attenzione i segnali formati dal verbo sapere (sai, sa, sapete), dire (dico, diciamo) e scusare (scusa, scusi, scusate). Il verbo sapere dà origine anche a diverse locuzioni verbali che vengono usate come $\mathrm{SD}$ : non so, che so; e così anche il verbo dire, da cui derivano le locuzioni: come dire, voglio dire, per cosi dire, a dire il vero, nonché intere clausole come te lo dico io. Va precisato che in questa sede saranno presi in considerazione solo i suddetti sintagmi verbali, e che le locuzioni verbali e le clausole saranno affrontate altrove. Resta anche inteso che, pur volendo essere più possibilmente comprensivi, non siamo in grado di offrire risposte definitive a proposito di questo concetto denominato talvolta "fuzzy" (Jucker \& Ziv, 1998: 2) o anche "sfuggente" (Bazzanella \& Borreguero Zuloaga, 2011: 8). 


\section{Quadro teorico}

In questo contributo si fa riferimento alla definizione ed alla tassonomia dei SD italiani fornite da C. Bazzanella (2008: 222) in chiave pragmatica, nelle quali l'autrice sottolinea la loro capacità di rivestire ruoli imporanti nella strutturazione del discorso e di segnalarne i rapporti tra le parti integranti nonché i processi cognitivi in corso, distinguendo le loro funzioni interazionali, metatestuali e cognitive (Tabelle 1, 2, 3 in Allegato).

Oltre alla polifunzionalità, illustrata quindi nelle apposite tabelle, ed alla ormai confermata alta frequenza dei SD nel parlato (Bazzanella, 1995; Frank-Job, 2006), molti studiosi (cfr. Schiffrin, 1987, 2001; Jucker \& Ziv, 1998; Fraser, 1999, 2006, 2009; Bazzanella, 1995, 2006) concordano in genere riguardo anche ad altri loro tratti, quali eterogeneità grammaticale, eliminabilità semantica, sensibilità al contesto, diversa collocazione, possibilità di cumulo (così come illustrato nel titolo del contributo). Per il processo interpretativo si rivela particolarmente importante la polifunzionalità sintagmatica, secondo la quale in un enunciato è possibile attribuire allo stesso SD più funzioni contemporaneamente, ad es. no? per la cessione del turno e la richiesta di conferma: Era bello, no? ('Bilo je lepo, zar ne?') (Bazzanella, 1995: 241, 2006, 2008), insieme ad un altro tratto, l'inter-sostituibilità (Bazzanella, 2001), che vede i SD spesso intercambiabili, a patto che svolgano la stessa funzione, come farebbe ad esempio al posto di no? il SD vero? in funzione di richiesta di conferma.

Ed è appunto il livello funzionale ovvero pragmatico (e non lessicale o semantico), che a seconda del contesto permette una pluralità di forme $\mathrm{e}$ funzioni svolte da SD, quello su cui va cercata la corrispondenza interlinguistica, il che rappresenta un compito non tanto facile per l'interprete se si considera che gli equivalenti semantici non sempre sono anche equivalenti a livello pragmatico, così come stabilito altrove (Ceković \& Radojević, 2016): in serbo, a differenza degli altri equivalenti semantici del segnale allora (dakle, onda, $p a, i$ ) si rivela che tada non può fungere anche da equivalente funzionale. Oppure ancora (Ceković \& Janićijević, in stampa), oltre all'equivalente semantico gledaj, si presentano anche čuj 'senti' o vidi 'vedi' come equivalenti pragmatici del segnale italiano guarda: Guarda non posso, non sto molto bene ('Čuj ne mogu, nisam baš dobro'). 


\section{Corpus}

Per il nostro studio contrastivo abbiamo estratto gli esempi da due romanzi di F. Moccia: Scusa, ma ti chiamo amore (AMO), Scusa, ma ti voglio sposare (SPO), e da un romanzo di N. Ammaniti: Io non ho paura (PAU) e le loro rispettive traduzioni in lingua serba. ${ }^{1}$ Nella ricerca ci siamo avvalsi del corpus elettronico dei testi letterari paralleli, parzialmente descritto in Moderc (2015). Abbiamo scelto i suddetti romanzi e le loro traduzioni in serbo poiché si tratta di opere letterarie contemporanee, ricche di dialoghi e monologhi interni che rispecchiano molte caratteristiche della lingua parlata e quindi offrono un contesto tipico in cui appaiono i SD, così come si può intuire già in base ai loro titoli.

\section{I SD dal verbo sapere}

Il SD sai (lo sai, sa, sapete) svolge funzioni pragmatiche di tipo interazionale. È caratterizzato da un'alta polifunzionalità paradigmatica, in quanto, in base alla posizione e all'intonazione, può assumere valori pragmatici diversi.

Sai (lo sai, sa, sapete) assolve generalmente il ruolo di fatismo e viene usato per sottolineare il rapporto interpersonale e la condivisione di conoscenze tra gli interlocutori. Questo suo uso è illustrato negli esempi (1)-(3):

- Carina la Smart, avrei voluto sempre - Sladak je „Smart“, uvek sam želela farmene una ma, sai, con due figli.... da imam jednog, ali, znaš, s dvoje (SPO) dece.. (UDA)

(2)

- Poi è innamorata da una vita del suo - A već sto godina je zaljubljena u svog ragazzo, sai, una di quelle storie eterne dečka, znaš, to je jedna od onih večitih che non capisci come fanno a durare priča za koje ne razumeš kako uspevaju da così tanto eppure durano (...). (SPO) traju tako dugo, pa ipak traju (...). (UDA)

$1 \quad$ Nelle citazioni si adoperano le sigle messe tra parentesi che rimandano ai romanzi da cui sono tratte. Per una visione completa del corpus si veda la Bibliografia alla fine del contributo. 


\section{(3)}

- Sì, ma sai, io sono ottimista di na- - Da, ali znaš, ja sam optimista po tura. Pensa che loro avevano detto che rođenju. Pomisli samo da su rekli da non saresti venuta, e invece... (AMO) nećeš doći, a ono... (LJUB)

Come si vede in (4)-(6), posto all'inizio di turno sai (lo sai, sa, sapete), può essere usato per richiamare l'attenzione e metterlo a conoscenza di un argomento sconosciuto.

(4)

Poi si ferma. Alza la testa. E guarda Onda se zaustavi. Digne glavu. I pogleuna stampa di Winnie the Pooh appesa da sliku Vini Pua na zidu. alla parete.

- Znaš, ostavila sam Roka... (UDA)

- Sai, ho lasciato Rocco... (SPO)

(5)

\begin{tabular}{|l|l|}
\hline Ha avuto un'esitazione. & Malo je oklevao. \\
- Lo sai, me lo avevano detto che tornavi. & - Znaš, rekli su mi da ćeš da se vratiti. \\
- Chi? (PAU) & - Ko? (BOJ)
\end{tabular}

(6)

Niki si accende una sigaretta e poi as- Niki zapali sebi jednu cigaretu i malo pira un po' (...). - Sai, a casa non posso uvuče dim (...). - Znaš, kod kuće ne mai fumare ... I miei non lo sanno che mogu nikad da pušim. Moji ne znaju da fumo. (AMO) pušim. (LJUB)

Posto, invece, alla fine dell'enunciato, il SD sai (lo sai, sa, sapete) serve a rafforzare un tono di stizza o una minaccia, un'intimazione:

(7)

- Certo però, Olly, mamma mia con - Ali, Oli, stvarno si dosadna s „tim "sto Giampi“ (...). Ne parli sempre! Đampijem" (...). Stalno pričaš o njeUna come te che diceva sempre male mu! I to ti, koja si uvek ružno pričala o dell'amore! Me lo ricordo, sai! (SPO) ljubavi! Znaš, sećam se ja! (UDA)

(8)

\begin{tabular}{|l|l|}
\hline - Sì, cerco te. T'ho scoperto, sai? Ho & - Da, tebe tražim. Otkrio sam te, znaš? \\
letto tutto. & Sve sam pročitao. \\
- Ma tutto che? Cosa vuoi da me? & - Ma šta to sve? Šta hoćeš od mene? \\
(SPO) & (UDA) \\
\hline
\end{tabular}


(9)

- E dai, non essere pesante. - Hajde, nemoj da si težak. Važno L'importante è averli visti ed evita- je da si ih video i izbegao, zar ne? ti, no? Magari devono andare a qual- Možda idu na neki važan sastanak... che appuntamento importante... $\quad$ - Da, onako obučeni.

- Sì, vestiti in quel modo. $\quad$ - Možda na neku audiciju. Moraju - Magari a un provino. Hanno biso- da rade. Nisu svi tatini sinovi, znaš? gno di lavorare. Mica sono tutti figli (LJUB) di papà, sai? (AMO)

Sai ha il suo equivalente serbo in znaš che svolge le stesse funzioni pragmatiche e in tutti i casi viene tradotto così. A nostro parere, però, esistono anche altre possibilità traduttive di questo SD in serbo. In (1) il suo equivalente pragmatico potrebbe essere anche znaš kako je 'sai com'è' o jasno ti je 'capisci' e in (2), tenendo conto della situazione linguistica, dell'età degli interlocutori e dell'argomento, al posto di znaš si potrebbe usare l'espressione colloquiale kapiraš 'capisci' o kontaš 'capisci'. In (7) e (8) sai potrebbe essere tradotto anche con l'espressione samo da znaš 'sappi', 'devi solo sapere', che può trovarsi sia all'inizio che alla fine della frase. Con questa espressione si otterrebbe un maggiore effetto di rafforzamento, solo che in (8) la frase non sarebbe più interrogativa, ma enunciativa.

\section{Il SD dal verbo dire}

Come abbiamo già menzionato, il verbo dire dà luogo a numerosi $\mathrm{SD}$, che vanno dai sintagmi verbali a intere frasi. In questa sede l'oggetto della nostra analisi saranno solo i sintagmi verbali dico e diciamo, in quanto, come osservato da Khachaturyan (2011: 111), proprio questi due SD del dire sono, in italiano, tra gli elementi più diffusi del parlato spontaneo.

\subsection{Il SD dico}

Il SD dico svolge una funzione metatestuale. Viene usato come focalizzatore e serve a sottolineare una parte dell'enunciato ovvero a mettere in evidenza un'informazione importante, la quale di norma precede questo SD. In (1), che si presta benissimo a illustrare questo suo uso, si riporta 
una conversazione tra madre e figlia dove il focalizzatore dico viene usato dalla madre proprio per ribadire il fatto che il fidanzato di sua figlia abbia 37 anni, cioè molti più di lei.

(1)

- Era venuto solo per farsi conoscere, - Došao je samo da vas upozna da biste così voi eravate un po' più tranquilli.... vi bili malo mirniji...

- E certo! Dopo questa bella notizia - Pa, naravno! Posle te lepe vesti bićemo saremo di un tranquillo che più tran- tako mirni da mirniji ne možemo biti... quillo non si può... Trentasette anni... Trideset sedam godina... Ne, ponavlNo dico, trentasette... (AMO) jam, trideset sedam... (LJUB)

(2) - Cioè, no. Secondo me, tu non ti rendi - U stvari, ne. Mislim da ti ne shvataš... conto... E voi? No, dico almeno voi ve A vi? Ne, mislim, barem vi to možete ne rendete conto o no? Questa è pazza! da shvatite, zar ne? Pa ova je luda! (AMO) (LJUB)

(3)

- A Ma', ma lo sai quanti provini ho - Mau, ma znaš li koliko sam audicija dovuto fare io prima d'essere presa a ja morala da idem pre nego što su me quello dell'altro giorno, eh? No dico, izabrali pre neki dan, a? Ne, stvarno, lo sai? (AMO) da li znaš? (LJUB)

L'equivalente semantico di dico è kažem il quale, però, non appare in nessuna delle traduzioni, dato che in serbo non può svolgere la stessa funzione pragmatica. Nelle traduzioni serbe, nella maggior parte dei casi, dico viene tradotto con mislim 'penso', che rappresenta il suo equivalente funzionale. In alcuni casi, illustrati qui da (1) e (3), sono state riscontrate altre traduzioni di questo SD, ma, a nostro parere, non tutte sono adeguate. Siamo dell'opinione che la traduzione serba di dico in (1) sia sbagliata (significa letteralmente ripeto) e pragmaticamente inadeguata. A nostro avviso, l'equivalente funzionale adatto anche in questo caso è mislim. Inoltre, come si vede, negli enunciati in italiano davanti a dico appare un "no" che aggiunge una sfumatura di sorpresa, fastidio. Questo "no" viene tradotto anche in serbo, ma ci risulta che il suo uso è superfluo, tranne in (3), dove si vede che l'equivalente pragmatico di dico, a seconda del contesto, può essere anche il segnale stvarno 'davvero'. 


\subsection{Il SD diciamo}

Come osserva Bazzanella (1995: 250), "diciamo è caratterizzato da un coinvolgimento fatico dell'interlocutore tramite l'uso della $1^{\mathrm{a}} \mathrm{ps}$. pl.“ e può svolgere diverse funzioni interazionali e metatestuali.

A livello interazionale viene usato come riempitivo e come meccanismo di modulazione con la funzione di mitigare il contenuto proposizionale dell'enunciato. In (1) sono contemporaneamente presenti entrambe le funzioni, quella di riempitivo, ma soprattutto di meccanismo di modulazione, in questo caso di attenuazione.

(1)

\begin{tabular}{|l|l|}
\hline - Ti sembra normale che & - Da li ti je normalno da si se \\
all’improvviso, praticamente senza & iznenada, praktično i ne poznajući \\
conoscerla, diciamo... ti sei divertito & je, da tako kažemo... zabavio \\
con la ragazza dei gelsomini? Con & sa devojkom jasmina? Sa Niki, \\
Niki, una diciassettenne. (AMO) & sedamnaestogodišnjakinjom. (LJUB) \\
\hline
\end{tabular}

Mentre l'esempio (1) illustra la polifunzionalità sintagmatica di dicia$m o$, in quanto nello stesso enunciato porta più valori contemporaneamente, l'esempio (2) ne illustra la polifunzionalità paradigmatica: accompagnato da -lo anaforico e generalmente pronunciato con volume alto, diciamo non ha più un uso attenuativo, ma assume una funzione diversa, quella di rafforzatore.

(2)

\begin{tabular}{|l|l|}
\hline - E la seconda è: perché non hai scelto & - A drugo: zašto mene nisi izabrao za \\
me come staff manager? & vođu tima? \\
- Perché Andrea Soldini me lo ha im- & - Zato što mi je Leonardo nametnuo \\
posto Leonardo. & Andreu Soldinija. \\
- Cioè è pure raccomandato! Ah, ecco, & - Znači, čak je i preko veze! Eto, pa \\
allora diciamolo, è pure un raccoman- & $\begin{array}{l}\text { dobro, recimo i to, on je preko veze. } \\
\text { dato. (AMO) }\end{array}$ \\
\hline
\end{tabular}

A livello metatestuale diciamo viene usato come indicatore di correzione, di maggiore precisione di un dato. Questo suo uso è illustrato in (3). 
SCUSA, SAI, TI DICO... GLI EQUIVALENTI SERBI DEI SEGNALI DISCORSIVI...

(3)

- Tu avevi bisogno di recuperare un po' - Tebi je bilo potrebno da povratiš malo di stima in te stesso, tranquillità e so- samopouzdanja, mir i pre svega... da prattutto... sfogo! Lui... bè, lui... era la daš sebi oduška! On, pa, on... to je bila sua festa... Cioè, più che festa diciamo njegova žurka... Pa, pre nego žurka, reil suo sacrificio! (SPO) cimo, njegovo žrtvovanje! (UDA)

L'equivalente serbo di diciamo è recimo, che è altresì indicatore di attenuazione, approssimazione e correzione, ma raramente un riempitivo. A seconda del contesto diciamo può avere anche altri equivalenti funzionali. Nell'esempio (1) lo troviamo tradotto con da tako kažemo 'diciamo così,' dove l'equivalente avrebbe potuto essere anche da tako kažem 'dico così', 'per così dire', visto che in serbo questa espressione è più frequente con il verbo alla $1^{\mathrm{a}} \mathrm{ps}$. sg. A nostro parere, invece, la traduzione in (2) non è adeguata. Il pronome lo è stato tradotto letteralmente e l'espressione recimo $i$ to non riesce a esprimere la stessa funzione di rafforzatore. È stato omesso il SD allora, che a nostro avviso, andava tradotto. Siamo del parere che gli equivalenti adeguati di diciamolo sarebbero in questo caso recimo onda 'diciamolo allora', recimo jasno i glasno 'diciamolo chiaro e tondo' ed eventualmente priznajmo onda 'ammettiamolo allora', i quali riuscirebbero a rafforzare meglio il contenuto proposizionale dell'enunciato.

Benché diciamo sia tra gli elementi più diffusi nel parlato spontaneo, spesso anche come indicatore di esemplificazione, ma soprattutto come riempitivo, possiamo constatare che le sue occorrenze nel nostro corpus sono abbastanza scarse. La scarsa frequenza di occorrenze è probabilmente dovuta al fatto che si tratta di opere scritte e, per quanto l'autore cerchi di usare un linguaggio simile al parlato, non è sempre facile riprodurre nello scritto un parlato spontaneo, una conversazione faccia a faccia in una situazione informale e poco controllata di cui è tipico l'uso di diciamo.

\section{Il SD dal verbo scusare}

Scusa (scusi, scusate) rientra tra iSD usati dalla parte dell'interlocutore e svolge la funzione interazionale di meccanismo di interruzione. Infatti, l'interlocutore lo usa per interrompere il parlante e per prendere il proprio turno: questa sua mossa viene spesso accompagnata dalla sovrapposizione dei loro enunciati. 
Gli esempi (1) e (2) dimostrano inoltre che nella presa di turno, quando è seguito da una domanda, scusa può funzionare contemporaneamente anche come richiesta di spegazione.

(1)

- T'intervisto semplicemente... che - Jednostavno ću razgovarati s tobom... orari puoi fare? Perché ho bisogno koje radno vreme ti odgovara? Pošto di qualcuno che stia con Ingrid quasi mi je potreban neko da bude $s$ Ingrid fino alle sette di sera... insomma, una do skoro sedam sati uveče... ukratko, certa elasticità.

potrebno mi je klizno radno vreme.

- Ma scusa... Questo non è un provino - Ali, izvini, zar ovo nije proba za per il ruolo di babysitter in un film? (SPO) ulogu bebisiterke u filmu? (UDA)

(2)

Flavio finisce di bere ed è come se gli Flavio dovrši piće i kao da mu je nešto venisse in mente qualcosa. palo na pamet.

- Ma scusa, quell'investigatore non - Izvini, a onaj detektiv pre dve goaveva trovato nulla due anni fa... gi- dine, ništa nije našao... je l' da? (UDA) usto? (SPO)

Come illustrato in (3) e (4) scusa viene usato anche all'inizio o alla fine di un enunciato in cui il parlante esprime un disaccordo, fastidio, un lieve rimprovero che cerca di attenuare un po' con scusa.

(3)

$-(\ldots)$, te l'ho detto mille volte... non mi $-(\ldots)$, hiljadu puta sam ti rekao... ne piace che vai da sola. (...). Cioè, non è sviđa mi se da ideš sama. (...). Odnosche non mi va che fai i provini, anzi, no, nije da mi se ne sviđa da ideš na ma mi piace proprio accompagnarti. audicije, naprotiv, ali baš volim da te - Ma scusa, guarda che nessuna delle ja otpratim.

altre viene accompagnata dal ragazzo. - Izvini, ali nijednu devojku ne prati (AMO) dečko. (LJUB)

(4)

\begin{tabular}{|l|l|}
\hline - Attenta, gli passi davanti. & - Pazi, prolaziš ispred njih. \\
- E che mi frega, scusa! (SPO) & - A šta me pa briga! (UDA) \\
\hline
\end{tabular}

A nostro avviso, il valore di disaccordo e fastidio è presente anche in (5) insieme a una sfumatura di stupore. 
SCUSA, SAI, TI DICO... GLI EQUIVALENTI SERBI DEI SEGNALI DISCORSIVI...

(5)

\begin{tabular}{l|l}
- Ma dove siamo? & -Ali, gde smo to mi?
\end{tabular}

- È una sorpresa! E sei anche bendata... - To je iznenađenje! A imaš i povez...

Ti pare che ti dico dove siamo?! Ma Zar misliš da bih ti rekao?! Ma daj, scusa, eh... Ecco, fermati qui. (SPO) molim te... Evo, stani tu. (UDA)

Per tradurre scusa, nella maggior parte dei casi, è stato usato il suo equivalente semantico izvini che assolve la stessa funzione pragmatica. In (5), però, il suo equivalente semantico non sarebbe pragmaticamente adeguato, a differenza dell'equivalente ma daj che appare nella traduzione. In (4), invece, scusa è omesso dalla traduzione, perché è davvero difficile trovare in questo caso un equivalente adeguato in senso pragmatico. Si può dire, tuttavia, che la traduzione sia riuscita, anche se non è stato possibile conservare appieno $\mathrm{i}$ valori di tipo emotivo che il SD italiano scusa veicola con sé.

\section{Conclusione}

In base a quanto esposto in questa sede, abbiamo dimostrato che $\mathrm{i}$ SD italiani derivanti dai verbi sapere, dire e scusare si possono trasmettere alquanto efficacemente in serbo. Come si è potuto vedere, nella maggioranza dei casi, i SD in questione sono stati tradotti con i loro equivalenti semantici che svolgono nello stesso tempo la medesima funzione pragmatica, mentre in alcuni sono stati completamente omessi oppure tradotti con i loro equivalenti funzionali. Si è riconfermato, però, che l'equivalenza semantica non implica necessariamente l'equivalenza funzionale (v. (5) Ma scusa, eh... 'Ma daj, molim te'), la quale, come già ribadito, risulta cruciale per la traduzione dei SD da una lingua all'altra. In altre parole, la corrispondenza interlinguistica tra i SD va sempre cercata a livello pragmatico.

Alla ricerca di corrispondenti pragmatici appunto, ci auguriamo pertanto che il presente lavoro, come anche gli altri che vi sono citati, possano rappresentare un utile punto di partenza e di riferimento per altre ricerche empiriche, basate sui corpora più vasti e incentrate su più SD diversi. Con questo studio abbiamo voluto dare il nostro contributo, sperando che esso possa trovare una sua applicazione anche nell'ambito della didattica contrastiva dell'italiano come lingua seconda o straniera. 


\section{Bibliografia}

Bazzanella, C. (1995). I segnali discorsivi. In L. Renzi, G. Salvi \& A. Cardinaletti (eds.), Grande grammatica italiana di consultazione, vol. 3 (pp. 225-257). Bologna: Il Mulino.

Bazzanella, C. (1999). Corrispondenze funzionali di well in italiano: analisi di un testo letterario e problemi generali. In G. Skytte \& F. Sabatini (eds.), Linguistica testuale comparativa. In memoriam Maria-Elisabeth Conte, Atti del Convegno interannuale, SLI, Copenhagen 5-7 febbraio 1998 (pp. 99-110). Copenhague: Museum Tusculanum Press.

Bazzanella, C. (2001). Segnali discorsivi e contesto. In W. Heinrich, C. Heiss \& M. Soffritti (eds.), Modalità e Substandard. Atti del Convegno Internazionale, Forli 26-27 ottobre 2000 (pp. 41-64). Bologna: CLUEB.

Bazzanella, C. (2006). Discourse markers in Italian: towards a "compositional" meaning. In K. Fischer (ed.), Approaches to Discourse Particles, vol. 1 (pp. 449-465). Oxford: Elsevier.

Bazzanella, C. (2008). Linguistica e pragmatica del linguaggio. Un'introduzione. Roma-Bari: Gius. Laterza \& Figli.

Bazzanella, C. \& Borreguero Zuloaga, M. (2011). 'Allora' e 'entonces': problemi teorici e dati empirici. Oslo Studies in Language, 3 (1), 7-45.

Bazzanella, C., Bosco, C., Garcea, A., Gili Fivela, B., Miecznikowski, J. \& Tini Brunozzi, F. (2007). Italian allora, French alors: Functions, convergences and divergences. Catalan Journal of Linguistics, 6, 9-30.

Blakemoore, D. (2002). Relevance and Linguistic Meaning: The Semantics and Pragmatics of Discourse Markers. Cambridge: Cambridge University Press.

Ceković, N. (2016). Diskursni markeri u govornoj produkciji na italijanskom kao drugom jeziku (Neobjavljena doktorska disertacija). Filološki fakultet, Beograd.

Ceković-Rakonjac, N. (2011). Različiti pristupi definisanju i opisu diskursnih markera. Anali Filološkog fakulteta, 23 (2), 133-153.

Ceković, N. \& Janićijević, N. (in stampa). La traduzione dei segnali discorsivi italiani in serbo: il caso dei verbi di percezione visiva e uditiva.

Ceković, N. \& Radojević, D. (2016). Polifunkcionalnost italijanskih diskursnih markera u prevođenju na srpski. In A. Vraneš \& Lj. Marković (eds.), Kulture u prevodu, knj. 2 (str. 233-246). Beograd: Filološki fakultet.

Deanović, M., \& Jernej, J. (1998). Talijansko-hrvatski rječnik. Zagreb: Školska knjiga.

Duro, A. (ed.) (2003). Il vocabolario Treccani. Sinonimi e contrari. Roma: Istituto dell'Enciclopedia italiana G. Treccani. 
Frank-Job, B. (2006). A dynamic-interactional approach to discourse markers. In K. Fischer (ed.), Approaches to Discourse Particles, vol. 1 (pp. 359-374). Oxford: Elsevier.

Fraser, B. (1998). Contrastive Discourse Markers in English. In A. Jucker \& Y. Ziv (eds.), Discourse markers: Description and Theory (pp. 301-326). Amsterdam/ Philadelphia: John Benjamins.

Fraser, B. (1999). What are discourse markers? Journal of Pragmatics, 31, 931-952.

Fraser, B. (2006). Towards a theory of discourse markers. In K. Fischer (ed.), Approaches to Discourse Particles, vol. 1 (pp. 189-204). Oxford: Elsevier.

Fraser, B. (2009). Topic orientation markers. Journal of Pragmatics, 41, 892-898.

Jucker, A. \& Ziv, Y. (eds.) (1998). Discourse markers: Description and Theory. Amsterdam/Philadelphia: John Benjamins.

Khachaturyan, E. (2010). The function of mitigation in spoken language. The analysis of "tak skazat" (Russian) and "diciamo" (Italian). Oslo Studies in Language, 2 (2), 393-412.

Khachaturyan, E. (2011). Una classificazione dei segnali discorsivi in italiano. Oslo Studies in Language, 3 (1), 95-116.

Khaciaturyan, E. (2002). Analisi contestuale dei segnali discorsivi. Indagine su diciamo. Studi Italiani di Linguistica Teorica e Applicata, 2, 245-258.

Klajn, I. (2011). Italijansko-srpski rečnik. Peto izmenjeno i dopunjeno izdanje. Beograd: Edicija.

Moderc, S. (2015). Su un modo di tradurre l'avverbio serbo "inače" in italiano: il caso dell'equivalente "altrimenti”. Italica Belgradensia, 2015 (1), 61-79.

Nigoević, M., \& Bilić M. (2009). Segnali discorsivi. Tempo guadagnato o tempo perduto. In Bastiansen, H. et al. (eds.), Tempo e memoria nella lingua e nella letteratura italiana, Atti del XVII Congresso A.I.P.I., vol. 1, (101113). Ascoli Piceno: Associazione Internazionale Professori d'Italiano.

Pittàno, G. (1997). Sinonimi e contrari. Dizionario fraseologico delle parole equivalenti, analoghe e contrarie. Bologna: Zanichelli.

Sabatini, F., \& Coletti, V. (2004). Dizionario della lingua italiana. Milano: Rizzoli - Larousse.

Schiffrin, D. (1987). Discourse Markers. Cambridge: Cambridge University Press.

Schiffrin, D. (2001). Discourse Markers: Language, Meaning, and Context. In Schiffrin, D., Hamilton, H., \& Tannen, D. (eds.), The Handbook of Discourse Analysis (54-75). Oxford: Blackwell.

Zingarelli, N. (1994). Lo Zingarelli. Vocabolario della lingua italiana. Bologna: Zanichelli.

Zingarelli, N. (2011). Lo Zingarelli. Vocabolario della lingua italiana. Bologna: Zanichelli. 
Nevena P. Ceković, Nataša L. Janićijević

\section{Fonti e sigle}

Amaniti, N. (2002). Ne bojim se (trad. D. Todorović - Lakava). Beograd: Plato. (BOJ)

Ammaniti, N (2001). Io non ho paura. Torino: Einaudi. (PAU)

Moća, F. (2008). Izvini, ali ti si moja ljubav (trad. B. Janković). Beograd: Čarobna knjiga. (LJUB)

Moća, F. (2011). Izvini, ali hoćeš li da se udaš za mene? (trad. B. Janković). Beograd: Čarobna knjiga. (UDA)

Moccia, F. (2007). Scusa ma ti chiamo amore. Milano: Rizzoli. (AMO)

Moccia, F. (2009). Scusa ma ti voglio sposare. Milano: Rizzoli. (SPO)

Nevena P. Ceković

Nataša L. Janićijević

\section{Sažetak}

\section{SCUSA, SAI, TI DICO... SRPSKI EKVIVALENTI ITALIJANSKIH DISKURSNIH MARKERA GLAGOLSKOG POREKLA}

Predmet rada predstavljaju italijanski diskursni markeri (DM) koji potiču od glagola sapere 'znati', dire 'reći' i scusare 'izviniti se' i njihovi srpski ekvivalenti. Teorijska osnova naše analize počiva na pragmatičkom pristupu DM, dok se jezički uzorak na kome je vršeno ispitivanje sastoji od književnih tekstova savremenih italijanskih autora i njihovih prevoda na srpski. Nakon sagledavanja osnovnih karakteristika i funkcija svakog pojedinačnog DM, u radu se izdvajaju i analiziraju njegovi prevodni ekvivalenti na srpskom jeziku. Rezultati analize ukazuju kako na semantičke tako i na pragmatičke ekvivalente na nivou dva jezika i potvrđuju zaključak da se korespondencija između DM u dva jezika utvrđuje na funkcionalnom nivou.

Ključne reči: italijanski diskursni markeri,srpski ekvivalenti, glagoli, prevođenje 


\section{Allegato}

Tabella I. Funzioni interazionali dei segnali discorsivi

(Bazzanella 2008)

\begin{tabular}{|l|l|}
\hline Funzioni interazionali & Dalla parte dell'interlocutore \\
\hline Dalla parte del parlante & 1. meccanismi di interruzione \\
\hline 1. presa di turno & 2. back-channels \\
2. "riempitivi" & 3. conferma dell'attenzione \\
3. richiesta di attenzione & 4. fatismi \\
4. fatismi & 5. - \\
5. meccanismi di "cortesia" & 6. acquisizione di conoscenza, richiesta \\
6. controllo della ricezione & di spiegazione \\
7. assunzione/richiesta di accordo & 7. accordo/conferma/rinforzo \\
e/o conferma & 8. - \\
8. cessione del turno & \\
\hline
\end{tabular}

Tabella II. Funzioni metatestuali dei segnali discorsivi

(Bazzanella 2008)

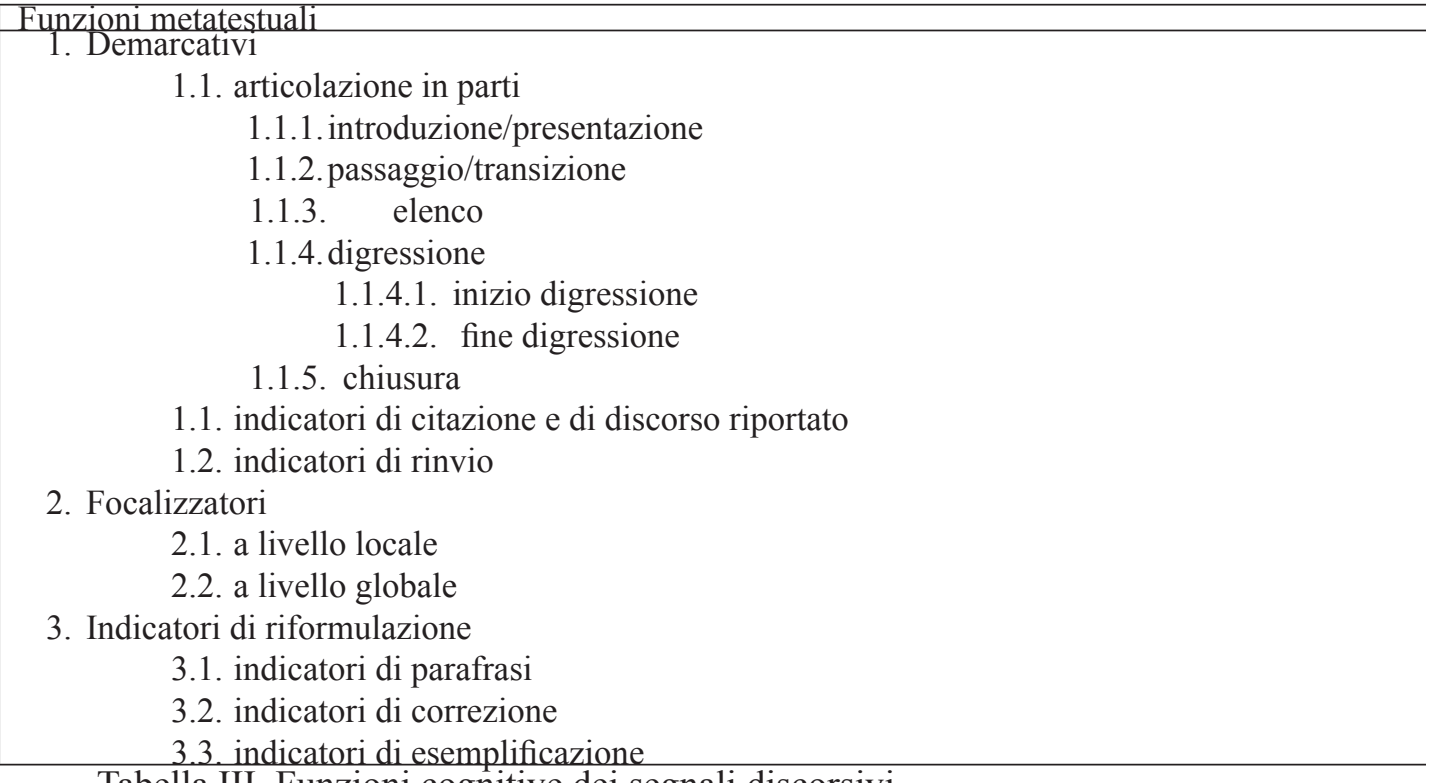

Tabella III. Funzioni cognitive dei segnali discorsivi

(Bazzanella 2008)

\begin{tabular}{|l|}
\hline Funzioni cognitive \\
\hline 1. Indicatori procedurali \\
2. Indicatori epistemici \\
3. Meccanismi di modulazione \\
\hline
\end{tabular}

